\title{
Forecasting the Daily Transaction Data Utilizing a Day of the Week Index in the Case of Web Site
}

\author{
Kazuhiro Takeyasu ${ }^{1} \&$ Tomohiro Watanabe ${ }^{2}$ \\ ${ }^{1}$ College of Business Administration, Tokoha University, Shizuoka City, Shizuoka, Japan \\ ${ }^{2}$ Dekita Inc, Fuji City, Shizuoka, Japan \\ Correspondence: Kazuhiro Takeyasu, College of Business Administration, Tokoha University, 6-1 Yayoicho \\ Suruga-ku, Shizuoka City, Shizuoka 422-8581, Japan.
}

Received: July 11, 2018

doi:10.5430/ijba.v9n5p11
Accepted: August 1, 2018

Online Published: August 7, 2018

\begin{abstract}
The daily web site access data's forecasting is an important factor for the web master in order to improve the quality of the site, and to increase the sales from the site. In this paper, the authors propose a new method to improve forecasting accuracy and confirm them by the Web transaction data (Nail consulting site). Focusing that the equation of exponential smoothing method (ESM) is equivalent to $(1,1)$ order ARMA model equation, a new method of estimation of smoothing constant in exponential smoothing method is proposed before by us which satisfies minimum variance of forecasting error. Generally, smoothing constant is selected arbitrarily. But in this paper, we utilize above stated theoretical solution. Firstly, the authors make estimation of ARMA model parameter and then estimate smoothing constants. Thus theoretical solution is derived in a simple way and it may be utilized in various fields. Combining the trend removing method with this method, we aim to improve forecasting accuracy. Furthermore, "a day of the week index" is newly introduced for the daily data and we have obtained good result. The effectiveness of this method should be examined in various cases.
\end{abstract}

Keywords: minimum variance, exponential smoothing method, forecasting, trend, web transaction data

\section{Introduction}

Correct sales forecasting is inevitable in industries. Poor sales forecasting accuracy leads to increased inventory and prolonged dwell time of product. In order to improve forecasting accuracy, we have devised trend removal methods as well as searching optimal parameters and obtained good results. We created a new method and applied it to various time series and examined the effectiveness of the method. Applied data are sales data, production data, shipping data, stock market price data, flight passenger data etc.

Many methods for time series analysis have been presented such as Autoregressive model (AR Model), Autoregressive Moving Average Model (ARMA Model) and Exponential Smoothing Method (ESM) (Box Jenkins, 1994), (R.G.Brown, 1963), (Tokumaru et al., 1982), (Kobayashi,1992). Among these, ESM is said to be a practical simple method.

For this method, various improving method such as adding compensating item for time lag, coping with the time series with trend (Peter, 1984), utilizing Kalman Filter (Maeda, 1984), Bayes Forecasting (M.West et al., 1989), adaptive ESM (Steinar, 1982), exponentially weighted Moving Averages with irregular updating periods (F.R.Johnston, 1993), making averages of forecasts using plural method (Spyros, 1983) are presented. For example, Maeda (1984) calculated smoothing constant in relationship with S/N ratio under the assumption that the observation noise was added to the system. But he had to calculate under supposed noise because he couldn't grasp observation noise. It can be said that it doesn't pursue optimum solution from the very data themselves which should be derived by those estimation. Ishii (1991) pointed out that the optimal smoothing constant was the solution of infinite order equation, but he didn't show analytical solution. Based on these facts, the authors proposed a new method of estimation of smoothing constant in ESM before (Takeyasu et al., 2008). Focusing that the equation of ESM is equivalent to $(1,1)$ order ARMA model equation, a new method of estimation of smoothing constant in ESM was derived.

In this paper, utilizing above stated method, a revised forecasting method is proposed. In making forecast such as 
Web transaction data (Nail consulting site), trend removing method is devised. Trend removing by the combination of linear and 2nd order non-linear function and 3rd order non-linear function is executed to the Web transaction data. The weights for these functions are varied by 0.01 increment and optimal weights are searched.

"a day of the week index (DWI)" is newly introduced for the daily data of Web transaction data(Nail consulting site) and a day of the week trend is removed. Theoretical solution of smoothing constant of ESM is calculated for both of the DWI trend removing data and the non DWI trend removing data. Then forecasting is executed on these data. This is a revised forecasting method. Variance of forecasting error of this newly proposed method is assumed to be less than those of the previously proposed method. The rest of the paper is organized as follows. In section 2, ESM is stated by ARMA model and estimation method of smoothing constant is derived using ARMA model identification. The combination of linear and non-linear function is introduced for trend removing in section 3. "a day of the week index (DWI)" is newly introduced in section 4. Forecasting is executed in section 5, and estimation accuracy is examined.

\section{Description of Esm Using Arma Model}

In ESM, forecasting at time $t+1$ is stated in the following equation.

$$
\begin{aligned}
\hat{x}_{t+1} & =\hat{x}_{t}+\alpha\left(x_{t}-\hat{x}_{t}\right) \\
& =\alpha x_{t}+(1-\alpha) \hat{x}_{t}
\end{aligned}
$$

Here,

$\hat{x}_{t+1}$ : forecasting at $t+1$

$x_{t}$ : realized value at $t$

$\alpha$ : smoothing constant $(0<\alpha<1)$

(1) is re-stated as:

$$
\hat{x}_{t+1}=\sum_{l=0}^{\infty} \alpha(1-\alpha)^{l} x_{t-l}
$$

By the way, we consider the following $(1,1)$ order ARMA model.

Generally, $(p, q)$ order ARMA model is stated as:

$$
x_{t}-x_{t-1}=e_{t}-\beta e_{t-1}
$$

$$
x_{t}+\sum_{i=1}^{p} a_{i} x_{t-i}=e_{t}+\sum_{j=1}^{q} b_{j} e_{t-j}
$$

Here,

$\left\{x_{t}\right\}$ : Sample process of Stationary Ergodic Gaussian Process $x(t) t=1,2, \cdots, N, \cdots$

$\left\{e_{t}\right\}$ : Gaussian White Noise with 0 mean $\sigma_{e}^{2}$ variance

MA process in (4) is supposed to satisfy convertibility condition.

Utilizing the relation that:

$$
E\left[e_{t} \mid e_{t-1}, e_{t-2}, \cdots\right]=0
$$

we get the following equation from (3).

$$
\hat{x}_{t}=x_{t-1}-\beta e_{t-1}
$$

Operating this scheme on $t+1$, we finally get: 


$$
\begin{aligned}
\hat{x}_{t+1} & =\hat{x}_{t}+(1-\beta) e_{t} \\
& =\hat{x}_{t}+(1-\beta)\left(x_{t}-\hat{x}_{t}\right)
\end{aligned}
$$

If we set $1-\beta=\alpha$, the above equation is the same with (1), i.e., equation of ESM is equivalent to $(1,1)$ order ARMA model, or is said to be $(0,1,1)$ order ARIMA model because 1st order AR parameter is -1 (Box Jenkins, 1994), (Tokumaru et al., 1982).

Comparing with (3) and (4), we obtain:

From (1), (6),

$$
\left\{\begin{array}{l}
a_{1}=-1 \\
b_{1}=-\beta
\end{array}\right.
$$

$$
\alpha=1-\beta
$$

Therefore, we get:

$$
\left\{\begin{array}{l}
a_{1}=-1 \\
b_{1}=-\beta=\alpha-1
\end{array}\right.
$$

From above, we can get estimation of smoothing constant after we identify the parameter of MA part of ARMA model. But, generally MA part of ARMA model become non-linear equations which are described below.

Let (4) be:

$$
\left\{\begin{array}{l}
\tilde{x}_{t}=x_{t}+\sum_{i=1}^{p} a_{i} x_{t-i} \\
\tilde{x}_{t}=e_{t}+\sum_{j=1}^{q} b_{j} e_{t-j}
\end{array}\right.
$$

The authors express the autocorrelation function of $\tilde{x}_{t}$ as $\tilde{r}_{k}$ and from (8), (9), we get the following non-linear equations which are well known ${ }^{[3]}$.

$$
\left.\begin{array}{lr}
\tilde{r}_{k}=\sigma_{e}^{2} \sum_{j=0}^{q-k} b_{j} b_{k+j} & (k \leq q) \\
0 & (k \geq q+1) \\
\tilde{r}_{0}=\sigma_{e}^{2} \sum_{j=0}^{q} b_{j}^{2} &
\end{array}\right\}
$$

For these equations, recursive algorithm has been developed. In this paper, parameter to be estimated is only $b_{1}$, so it can be solved in the following way.

From (3) (4) (7) (10), we get:

$$
\left.\begin{array}{l}
q=1 \\
a_{1}=-1 \\
b_{1}=-\beta=\alpha-1 \\
\widetilde{r}_{0}=\left(1+b_{1}^{2}\right) \sigma_{e}^{2} \\
\widetilde{r}_{1}=b_{1} \sigma_{e}^{2}
\end{array}\right\}
$$


If we set:

$$
\rho_{k}=\frac{\widetilde{r}_{k}}{\widetilde{r}_{0}}
$$

the following equation is derived.

$$
\rho_{1}=\frac{b_{1}}{1+b_{1}^{2}}
$$

We can get $b_{1}$ as follows.

$$
b_{1}=\frac{1 \pm \sqrt{1-4 \rho_{1}^{2}}}{2 \rho_{1}}
$$

In order to have real roots, $\rho_{1}$ must satisfy:

$$
\left|\rho_{1}\right| \leq \frac{1}{2}
$$

From invertibility condition, $b_{1}$ must satisfy:

From (13), using the next relation,

$$
\left|b_{1}\right|<1
$$

$$
\begin{aligned}
& \left(1-b_{1}\right)^{2} \geq 0 \\
& \left(1+b_{1}\right)^{2} \geq 0
\end{aligned}
$$

(15) always holds.

As

$b_{1}$ is within the range of:

$$
\alpha=b_{1}+1
$$

Finally we get:

$$
-1<b_{1}<0
$$

$$
\left.\begin{array}{l}
b_{1}=\frac{1-\sqrt{1-4 \rho_{1}^{2}}}{2 \rho_{1}} \\
\alpha=\frac{1+2 \rho_{1}-\sqrt{1-4 \rho_{1}^{2}}}{2 \rho_{1}}
\end{array}\right\}
$$

which satisfy above condition. Thus we can obtain a theoretical solution by a simple way.

Here $\rho_{1}$ must satisfy:

$$
-\frac{1}{2}<\rho_{1}<0
$$

in order to satisfy $0<\alpha<1$.

Focusing on the idea that the equation of ESM is equivalent to $(1,1)$ order ARMA model equation, the authors can estimate smoothing constant after estimating ARMA model parameter.

It can be estimated only by calculating 0 th and 1 st order autocorrelation function.

\section{Trend Removal Method}

As trend removal method, the authors describe the combination of linear and non-linear function. 
[1] Linear function

We set:

$$
y=a_{1} x+b_{1}
$$

as a linear function.

[2] Non-linear function

We set:

$$
\begin{gathered}
y=a_{2} x^{2}+b_{2} x+c_{2} \\
y=a_{3} x^{3}+b_{3} x^{2}+c_{3} x+d_{3}
\end{gathered}
$$

as a $2^{\text {nd }}$ and a $3^{\text {rd }}$ order non-linear function.

[3] The combination of linear and non-linear function

We set:

$$
\begin{aligned}
& y=\alpha_{1}\left(a_{1} x+b_{1}\right)+\alpha_{2}\left(a_{2} x^{2}+b_{2} x+c_{2}\right) \\
& +\alpha_{3}\left(a_{3} x^{3}+b_{3} x^{2}+c_{3} x+d_{3}\right) \\
& 0 \leq \alpha_{1} \leq 1 \quad, 0 \leq \alpha_{2} \leq 1 \quad, 0 \leq \alpha_{3} \leq 1 \\
& \alpha_{1}+\alpha_{2}+\alpha_{3}=1
\end{aligned}
$$

as the combination of linear and $2^{\text {nd }}$ order non-linear and $3^{\text {rd }}$ order non-linear function.

Trend is removed by dividing the data by (21).

\section{A Day of the Week Index}

"a day of the week index (DWI)" is newly introduced for the daily Web transaction data. The forecasting accuracy would be improved after we identify the "a day of the week index" and utilize them. This time in this paper, the data we handle consist by Monday through Sunday, The authors calculate $\operatorname{DWI}_{j}(j=1, \cdots, 7)$ for Monday through Sunday.

For example, if there is the daily data of $L$ weeks as stated bellow:

$$
\left\{x_{i j}\right\}(i=1, \cdots, L)(j=1, \cdots, 7)
$$

where $x_{i j} \in R$ in which $L$ means the number of weeks (Here $L=10$ ), $i$ means the order of weeks ( $i$-th week), $j$ means the order in a week ( $j$-th order in a week; for example $j=1:$ Monday, $j=7:$ Sunday) and $x_{i j}$ is the daily Web transaction data (Nail consulting site). Then, $D W I_{j}$ is calculated as follows.

$$
D W I_{j}=\frac{\frac{1}{L} \sum_{i=1}^{L} x_{i j}}{\frac{1}{L} \cdot \frac{1}{7} \sum_{i=1}^{L} \sum_{j=1}^{7} x_{i j}}
$$

DWI trend removal is executed by dividing the data by (23). Numerical examples both of DWI removal case and non-removal case are discussed in section 5. 


\section{Forecasting the Daily Web Transaction Data}

\subsection{Analysis Procedure}

The daily Web transaction data (Nail consulting site) of the following 2 cases are analyzed.

Term 1: January 1, 2016 to March 24, 2016

Term 2: October 1, 2016 to December 23, 2016

First of all, graphical charts of these time series data are exhibited in Figures 1 and 2.

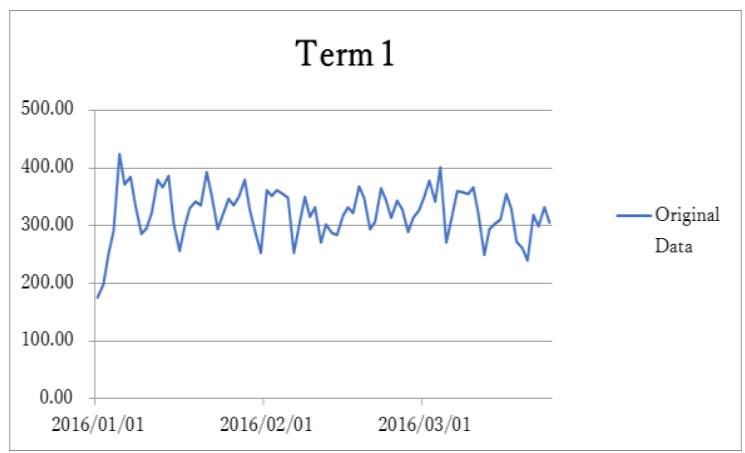

Figure 1. Daily data of term 1

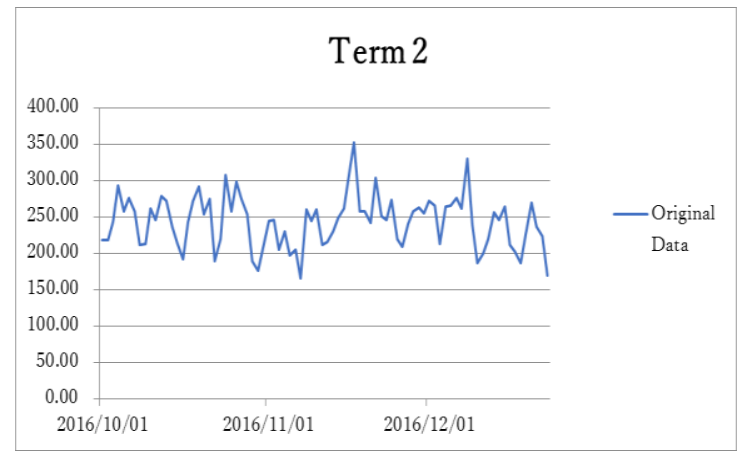

Figure 2. Daily data of term 2

(2016.1-2016.3)

(2016.10-2016.12)

Analysis procedure is as follows. There are 84 daily data for each case. The authors use 70 data ( 1 to 70 ) and remove trend by the method stated in section 3. Then the authors calculate a day of the week index (DWI) by the method stated in section 4. After removing DWI trend, the method stated in section 2 is applied and Exponential Smoothing Constant with minimum variance of forecasting error is estimated. Then 1 step forecast is executed. Thus, data is shifted to 2nd to 51 st and the forecast for 72 nd data is executed consecutively, which finally reaches forecast of 84th data. To examine the accuracy of forecasting, variance of forecasting error is calculated for the data of 71st to 84th data. Final forecasting data is obtained by multiplying DWI and trend.

Forecasting error is expressed as:

$$
\begin{gathered}
\varepsilon_{i}=\hat{x}_{i}-x_{i} \\
\bar{\varepsilon}=\frac{1}{N} \sum_{i=1}^{N} \varepsilon_{i}
\end{gathered}
$$

Variance of forecasting error is calculated by:

$$
\sigma_{\varepsilon}^{2}=\frac{1}{N-1} \sum_{i=1}^{N}\left(\varepsilon_{i}-\bar{\varepsilon}\right)^{2}
$$

In this paper, the authors examine the two cases stated in Table 1.

Table 1. The combination of the case of trend removal and DWI trend removal

\begin{tabular}{ccc}
\hline Case & Trend & DWI trend \\
\hline Case1 & Removal & Removal \\
Case2 & Removal & Non removal \\
\hline
\end{tabular}

\subsection{Trend Removing}

Trend is removed by dividing original data by (21). Here, the weight of $\alpha_{1}$ and $\alpha_{2}$ are shifted by 0.01 increment 
in (21) which satisfy the equation (22). The best solution is selected which minimizes the variance of forecasting error. Estimation results of coefficient of (18), (19) and (20) are exhibited in Table 2. Data are fitted to (18), (19) and (20), and using the least square method, parameters of (18), (19) and (20) are estimated. Estimation results of weights of (21) are exhibited in Table 3. The weighting parameters are selected so as to minimize the variance of forecasting error.

Table 2. Coefficient of (18),(19) and (20)

\begin{tabular}{lccrrrrrrr}
\hline & \multicolumn{1}{c}{$\mathbf{1}^{\text {st }}$} & \multicolumn{1}{c}{$\mathbf{2}^{\text {nd }}$} & \multicolumn{4}{c}{$\mathbf{3}^{\text {rd }}$} \\
& $a_{1}$ & \multicolumn{1}{c}{$b_{1}$} & \multicolumn{1}{c}{$a_{2}$} & \multicolumn{1}{c}{$b_{2}$} & \multicolumn{1}{c}{$c_{2}$} & $a_{3}$ & \multicolumn{1}{c}{$b_{3}$} & \multicolumn{1}{c}{$c_{3}$} & $d_{3}$ \\
\hline Term 1 & 0.40 & 311.89 & -0.01 & 0.82 & 306.85 & 0.00 & -0.26 & 8.21 & 261.58 \\
Term 2 & 0.28 & 237.97 & 0.02 & -1.00 & 253.32 & 0.00 & 0.00 & -0.39 & 249.57 \\
\hline
\end{tabular}

Table 3. Weights of (21)

\begin{tabular}{ccccc}
\hline & Case & $\alpha_{1}$ & $\alpha_{2}$ & $\alpha_{3}$ \\
\hline \multirow{2}{*}{ Term 1 } & Case1 & 1.00 & 0.00 & 0.00 \\
& Case2 & 1.00 & 0.00 & 0.00 \\
\multirow{2}{*}{ Term 2 } & Case1 & 0.00 & 1.00 & 0.00 \\
& Case2 & 1.00 & 0.00 & 0.00 \\
\hline
\end{tabular}

As a result, we can observe the following three patterns.

(1) Selected $1^{\text {st }}$ order model:

Term 1 Case1, Case2, Term 2 Case2

(2) Selected 2nd order model:

Term 2 Case 1

Graphical charts of trend are exhibited in Figures 3 to 4.

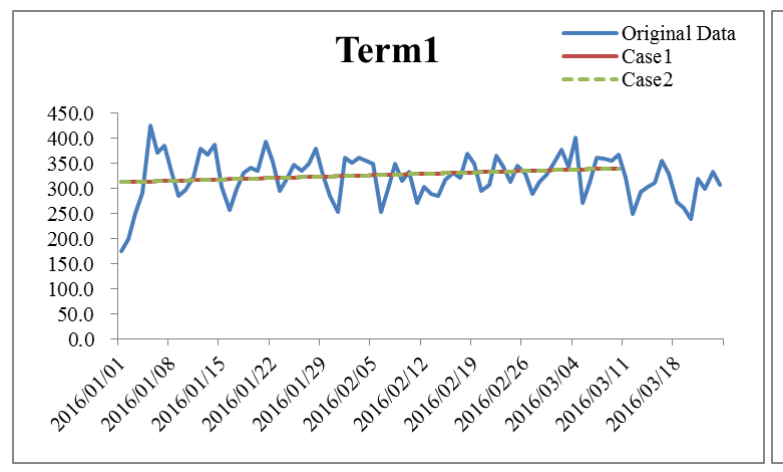

Figure 3. Daily data of term 1

(2016.1-2016.3)

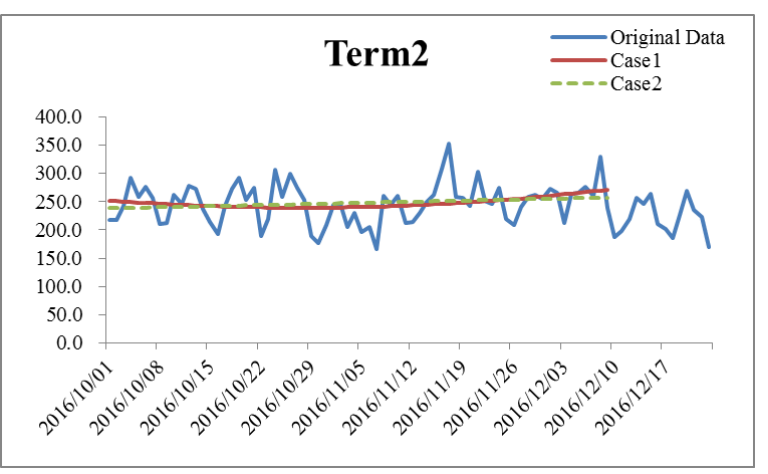

Figure 4. Daily data of term 2

(2016.10-2016.12)

\subsection{Removing Trend by DWI}

After removing trend, a day of the week index is calculated by the method stated in 4. Calculation result for 1st to 70th data is exhibited in Table 4. 
Table 4. A Day of the week index

\begin{tabular}{|c|c|c|c|c|c|c|c|c|}
\hline \multirow{2}{*}{ Term } & \multirow{2}{*}{ Case } & \multicolumn{7}{|c|}{ a day of the week index } \\
\hline & & Thu. & Fri. & Sat. & Sun. & Mon. & Tue. & Wed. \\
\hline 1 & Case1 & 0.988 & 0.834 & 0.899 & 1.033 & 1.082 & 1.066 & 1.098 \\
\hline 2 & Case1 & 0.855 & 0.888 & 1.012 & 1.060 & 1.086 & 1.114 & 0.985 \\
\hline
\end{tabular}

\subsection{Estimation of Smoothing Constant With Minimum Variance of Forecasting Error}

After removing DWI trend, Smoothing Constant with minimum variance of forecasting error is estimated utilizing (16). There are cases that we cannot obtain a theoretical solution because they do not satisfy the condition of (17). In those cases, Smoothing Constant with minimum variance of forecasting error is derived by shifting variable from 0.01 to 0.99 with 0.01 interval. Calculation result for 1 st to 70 th data is exhibited in Table 5 .

Table 5. Estimated smoothing constant with minimum variance

\begin{tabular}{cccc}
\hline & Case & $\rho_{1}$ & $\alpha$ \\
\hline \multirow{2}{*}{ Term 1 } & Case1 & -0.4086 & 0.482 \\
& Case2 & -0.1491 & 0.847 \\
\multirow{2}{*}{ Term 2 } & Case1 & -0.6985 & 0.030 \\
& Case2 & -0.2802 & 0.693 \\
\hline
\end{tabular}

\subsection{Forecasting and Variance of Forecasting Error}

Utilizing smoothing constant estimated in the previous section, forecasting is executed for the data of 71st to 84th data. Final forecasting data is obtained by multiplying DWI and trend. Variance of forecasting error is calculated by (26).

Forecasting results are exhibited in Figures 5 and 6.

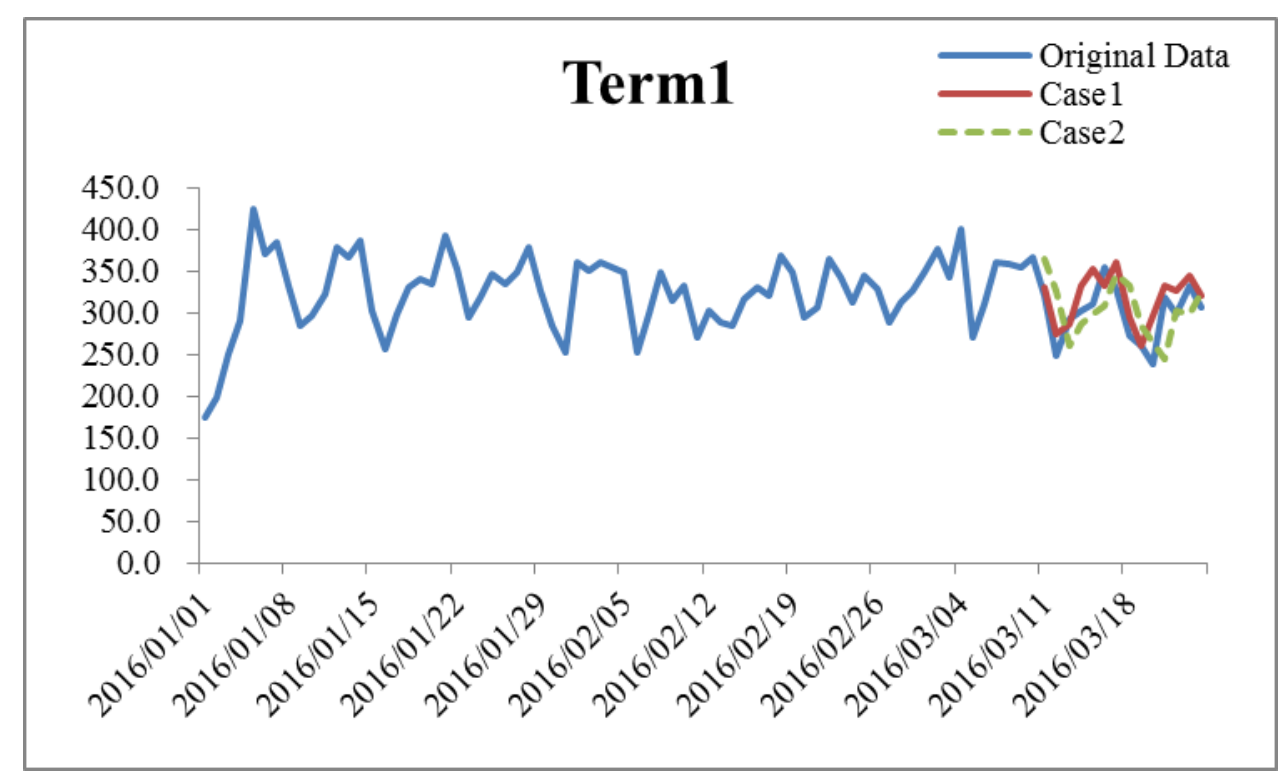

Figure 5. Forecasting results of term 1 


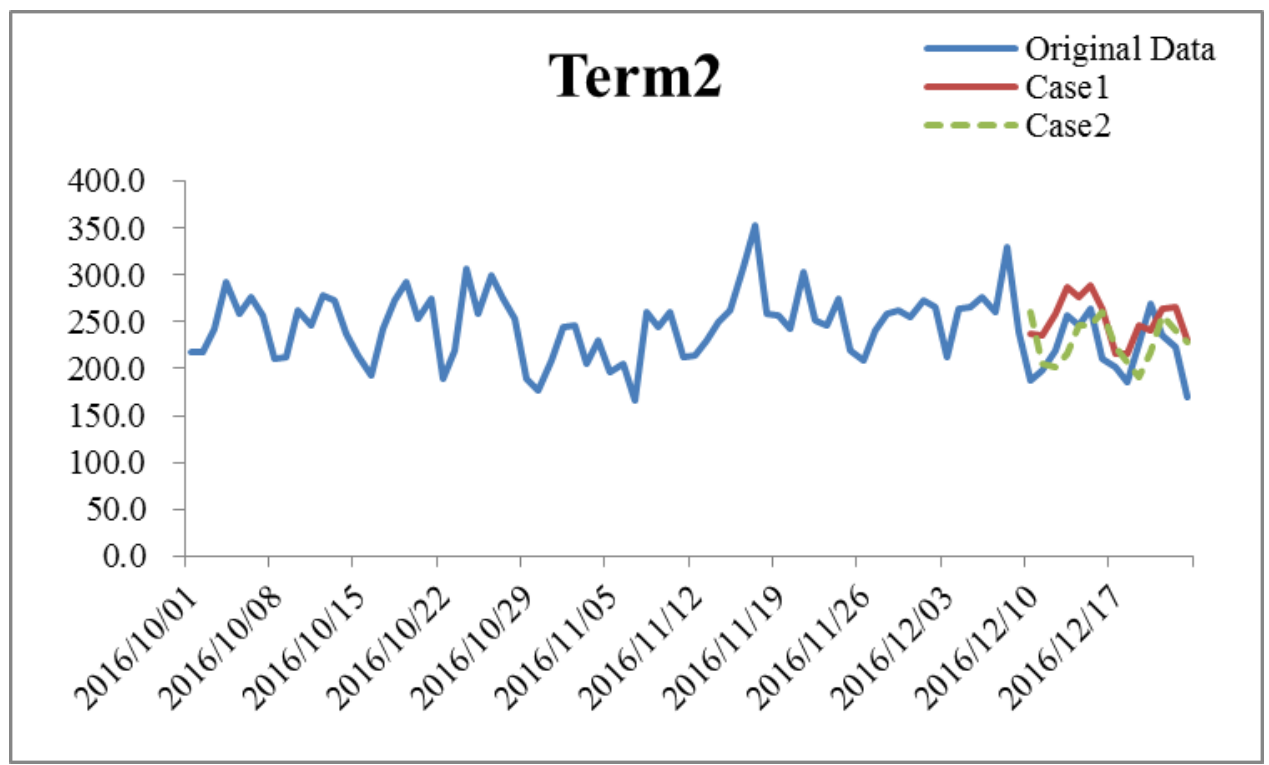

Figure 6. Forecasting results of term 2

Variance of forecasting error is exhibited in Table 6.

Table 6. Variance of forecasting error

\begin{tabular}{lcrr}
\hline & Case & Variance of Forecasting Error & \\
\hline Term 1 & Case1 & 218 & $*$ \\
& Case2 & 534 & \\
Term 2 & Case1 & 172 & $*$ \\
& Case2 & 446 & \\
\hline
\end{tabular}

\section{Conclusions}

Correct sales forecasting is inevitable in industries. Focusing on the idea that the equation of exponential smoothing method(ESM) was equivalent to $(1,1)$ order ARMA model equation, a new method of estimation of smoothing constant in exponential smoothing method was proposed before by us which satisfied minimum variance of forecasting error. Generally, smoothing constant was selected arbitrarily. But in this paper, the authors utilized above stated theoretical solution. Firstly, the authors made estimation of ARMA model parameter and then estimated smoothing constants. Thus theoretical solution was derived in a simple way and it might be utilized in various fields.

Furthermore, combining the trend removal method with this method, the authors aimed to increase forecasting accuracy. An approach to this method was executed in the following method. Trend removal by a linear function was applied to the daily data of Web transaction data (Nail consulting site). The combination of linear and non-linear function was also introduced in trend removing. "a day of the week index (DWI)" is newly introduced for the daily data and a day of the week trend is removed. Theoretical solution of smoothing constant of ESM was calculated for both of the DWI trend removing data and the non DWI trend removing data. Then forecasting was executed on these data.

Regarding both data, the forecasting accuracy of case1 (DWI is imbedded) was better than those of case 2 (DWI is not imbedded). It can be said that the introduction of DWI has worked well. It is our future works to ascertain our newly proposed method in many other cases. The effectiveness of this method should be examined in various cases.

\section{References}

Box Jenkins. (1994). Time Series Analysis (3rd ed.). Prentice Hall.

Brown, R.G. (1963). Smoothing, Forecasting and Prediction of Discrete-Time Series. Prentice Hall.

Ekern, S. (1982). Adaptive Exponential Smoothing Revisited. Journal of the Operational Research Society, 32 , 
775-782. https://doi.org/10.1057/jors.1981.155

Ishii, N. et al. (1991). Bilateral Exponential Smoothing of Time Series. Int.J.System Sci., 12(8), 997-988. https://doi.org/10.1287/mnsc.29.9.987

Johnston, F.R. (1993). Exponentially Weighted Moving Average (EWMA) with Irregular Updating Periods. Journal of the Operational Research Society, 44(7), 711-716. https://doi.org/10.1057/jors.1993.120

Kobayashi, K. (1992). Sales Forecasting for Budgeting. Chuokeizai-Sha Publishing.

Maeda, K. (1984). Smoothing Constant of Exponential Smoothing Method. Seikei University Report Faculty of Engineering No. 38, 2477-2484.

Makridakis, S., \& Winkler, R.L. (1983). Averages of Forecasts; Some Empirical Results. Management Science, 29(9), 987-996.

Peter R.Winters. (1984). Forecasting Sales by Exponentially Weighted Moving Averages. Management Science, 6(3), 324-343. https://doi.org/10.1287/mnsc.6.3.324

Takeyasu, K., \& Nagao, K. (2008). Estimation of Smoothing Constant of Minimum Variance and its Application to Industrial Data. Industrial Engineering and Management Systems, 7(1), 44-50.

Tokumaru, H. et al.. (1982). Analysis and Measurement-Theory and Application of Random data Handling. Baifukan Publishing.

West, M., \& Harrison, P.J. (1989). Baysian Forecasting and Dynamic Models. Springer-Verlag, New York. https://doi.org/10.1007/978-1-4757-9365-9 